\title{
Oral Health Knowledge, Practice, Oral Hygiene Status and Dental Caries Prevalence Among Visually Impaired Student in Alnoor Institute of Almadinah Almunawwarah at Saudi Arabia
}

\author{
Azzah Alhazmi ${ }^{1}$, Ishraq Alshinqiti ${ }^{1}$, Fayez Aloufi ${ }^{1}$, Abdulmoeen Alhejali ${ }^{2}$, Waleed Alsulaimani ${ }^{3, *}$, \\ Mohammed Alharbi ${ }^{4}$ \\ ${ }^{1}$ Previous Dental intern, Speciality Dental Center of King Fahad Hospital, Almadinah, Saudi arabia \\ ${ }^{2}$ Acting Consultant in Oral Surgery, Speciality Dental Center of King Fahad Hospital, Almadinah, Saudi arabia \\ ${ }^{3}$ Consultant Orthodontist, Speciality Dental Center of King Fahad Hospital, Almadinah, Saudi arabia \\ ${ }^{4}$ Consultant Prosthodontist, Speciality Dental Center of King Fahad Hospital, Almadinah, Saudi arabia \\ *Corresponding author: dr.waleed.s@hotmail.com
}

Received November 15, 2014; Revised November 21, 2014; Accepted November 25, 2014

\begin{abstract}
Aim: to investigate the oral health knowledge, practice, oral hygiene status and the prevalence of dental caries among visually impaired student in Alnoor institute of Almadinah, Saudi Arabia. Material and methods: the study population consisted of 64 visually impaired student attending Alnoor institute in Almadinah in the age group of (7-24) years old. Interview questionnaires were used to assess oral health knowledge and practice through. The simplified oral hygiene index was used to assess oral hygiene and DMFT/dmft for caries prevalence. Result: the study revealed $4.84 \%$ of the students were free of caries while caries prevalence is $95.16 \%$ which is very high and coincide when comparing it to control group from other study in the same area. The mean of DMFT/dmft is low $0.24 / 0.59$ and the reason for that may be various, ranging from biochemical differences in salivary buffering to differences in living environment, dietary and hygiene habits. This study revealed insignificant between neither the mean of DMFT/dmft for both gender Male \& Female 0.24/0.57 and 0.24/0.65 nor the mean of plaque index 1.5/1.33 respectively. Also the age group distribution was insignificant. Among Survey 93.7\% agree with role of sugar in leading to caries while $3.1 \%$ disagree \& $3.1 \%$ they don't know. There is $85.9 \%$ do brushing of their teeth and $14.1 \%$ they don't. There is $87.5 \%$ agree with that brushing will prevent caries but $9.47 \%$ they disagree. For the method of brushing it was found that $93.2 \%$ use tooth brush $3.4 \%$ use fingers and 3.4\% use meswak. Conclusion: the finding of this study revealed high caries prevalence and low DMFT/dmft and more than 2/3 of the sample have equal or greater than 1-2 soft debris accumulation. A little extra care by the parent or caretaker regarding oral hygiene can give drastic result in reduction of dental caries and gingival disease.
\end{abstract}

Keywords: oral hygiene, prevalence of dental caries, visually impaired

Cite This Article: Azzah Alhazmi, Ishraq Alshinqiti, Fayez Aloufi, Abdulmoeen Alhejali, Waleed Alsulaimani, and Mohammed Alharbi, "Oral Health Knowledge, Practice, Oral Hygiene Status and Dental Caries Prevalence Among Visually Impaired Student in Alnoor Institute of Almadinah Almunawwarah at Saudi Arabia.” International Journal of Dental Sciences and Research, vol. 2, no. 6 (2014): 149-153. doi: 10.12691/ijdsr-2-6-7.

\section{Introduction}

Blindness is defined by World Health Organization as having a: "visual acuity of less than $3 / 60 \mathrm{~m}$ or corresponding visual field loss in the better eye with the best possible correction ", meaning that whilst a blind person could see a distance of $3 \mathrm{~m}$, a non-visually impaired person could see $60 \mathrm{~m}$. Visual impairment relates to a person's eyesight which cannot be corrected to normal vision.

The WHO estimates that there are 40 million blind persons in the world. Visually impaired individuals cannot visualize the plaque on the teeth surface so even understanding the importance of oral hygiene is difficult for them which result in progression of dental caries as well as inflammatory disease of the periodontium (Mann et al, 1977) [1].

Dental caries or periodontal disease are not a priority of the disable student's families (Tesini'1981; cutron, 1971) [2].

Oral health education has been shown to have a positive impact in lowering plaque score (Zehaati and Motlagh, 2006) [3].

There is little information available regarding the dental health care and needs of such individuals. Such information is important to improve the oral health in these special need children. The aim of this study was to 
investigate the oral health knowledge, practice, oral hygiene status and the prevalence of dental caries among blind student in Alnoor institute of Almadinah, Saudi Arabia.

\section{Materials and Methods}

\subsection{Study Population and Sample Design}

The study was conducted among Alnoor institute for females and males and the public intermediate school which included 4 female girls. A total no of 80 (51 male 29 female) were reached. The sampling technique was convenience. All blind children attending the governmental schools "male and female" from 1st to 9th grade were included. Any child with combined disability was excluded. The study was carried out on a 7 - 24 aged students. An interview questionnaire was done among students in the classroom. The clinical exam forms were attached to the questionnaires. Each student keeps the questionnaire with him/her for the examiner to record the clinical exam on the same form.

\subsection{Measures and Definitions}

- DMFT and dmft index: Most of the students were in the age of mixed dentition $(6-18)$. The DMFT and $\mathrm{dmft}$ were used to measure the prevalence of dental caries/teeth. Each child is given a separate index, one for permanent teeth and another for primary teeth.

- Plaque and calculus index: the simplified oral hygiene index was used to record the plaque in six teeth surfaces. The six teeth are:

Maxillary right first molar "buccally", right first incisor "labialy", left first molar "buccaly".

Mandibular right first molar "lingually", left first incisor "labially", left first molar "lingually".

Criteria for scoring:

$0=$ no debris or stain.

$1=$ soft debris covering not $>1 / 3$ of tooth OR the presence of extrinsic stains regard less of surface area covered.

$2=$ soft debris covering $>1 / 3$ but not $>2 / 3$ of tooth surface. $3=$ soft debris covering $>2 / 3$ of tooth surface.

DI - S = total debris score / no. of teeth examined.

The visually impaired children were examined at their respective schools, seated on an ordinary chair, and sharp metallic prop and metallic mirror were used.

\section{- Dental health knowledge}

Through the interview the student were asked if tooth brushing prevent caries, bleeding, and halitosis. They were also asked if sweets have a role in tooth caries. The students were asked generally if they knew if florid has an advantage on their teeth or not. According to the student answers on where did they learn to brush their teeth, they were three groups, from school, television, and home.

- Oral health status and Past dental visit:

The interview questionnaire included questions about the oral health status, the mode of tooth cleaning, the substance used for cleaning teeth, when and who help if needed, the use of dentifrices, the use of dental floss and the tooth cleaning frequency. Based on a question on past dental visits, the sample was divided into four groups, namely students who reported through the interview that they had never visited a dentist before, students who had visited the dentist in the past 6 months, students who have been to the dentist in more than 6 months and finally those who had visited a dentist since more than one year. The reason behind seeking the dentist was also assessed for check - ups or pain only.

\section{- General oral examination:}

All school students received a general oral examination to identify their need for oral/dental health but not intended to be diagnostic. The examiner looked for plaque, gingivitis, caries and any oral mucosal lesions.

\section{- Operational design:}

After a preliminary visit to the school was conducted, the data was collected through a double field trip to the female primary school by the intern researchers and the female intermediate school. The male institute included the student from grade 1 to 9 ; a double visit was done to them by the intern researcher and a helping friend to write down the data.

An educational health program was done to the school students through oral short story, pamphlets and models. Gifts were also distributed to them supplied from the SDS almedinah.

\section{- Administrative and ethical design:}

Approval was granted by the Minister of Education. A written Consent was distributed through the student to be signed by the caregiver. Following the oral examination, an examination card was given to each student to indicate which dental - related services the student might need. Confidentiality of response result and to avoid response bias, teachers were asked not to enter the classroom during both questionnaire administration and clinical examination.

- Data analysis:

The data analysis was carried out by SPSS software version 16. Descriptive statistics as mean, standard deviation and proportion will be used as indicated.

\section{Result}

A total of 80 visually impaired children from Alnoor institute in Almadinah, KSA. Upon the field trips, 4 children were absents and one student refused to get the examination. With the exclusion of 11 student which had multiple disability, a remaining of 64 child were examined of (DMFT - dmft - green \&vermillion Plaque index) (Table 1) and had interview questionnaire. Out of total there were 42 (66\%) Males and 22 (34\%) Females, all in the age group of (7-24) years old. Maximum numbers were collected of 10 \& 15 years of age and overall mean of age was 12.1 years. Each child had interview questionnaire regarding oral hygiene practice, attitude and knowledge.

\begin{tabular}{|c|c|c|c|c|c|}
\hline & $\mathrm{N}$ & Minimum & Maximum & Mean & $\begin{array}{c}\text { Std. } \\
\text { deviation }\end{array}$ \\
\hline DMFT & 60 & .00 & .53 & .2370 & .13756 \\
\hline $\mathrm{dmft}$ & 37 & .00 & 1.00 & .5943 & .35046 \\
\hline $\begin{array}{c}\text { Plaque Index } \\
\text { G\&V }\end{array}$ & 62 & .37 & 2.66 & 1.4402 & .48266 \\
\hline
\end{tabular}

Caries prevalence revealed $95.16 \%$ of the visually impaired children have Caries. The mean of DMFT/dmft 
in Males was 0.24/0.57 and 0.24/0.65 in Females. Plaque index was 1.5 in Males and 1.33 in Females. While the mean of DMFT/dmft in elementary school level $(n=50$ students) was $0.23 / 0.56$ and $0.28 / 0.8$ in middle school level ( $\mathrm{n}=14$ students). Plaque index was 1.3 in elementary school level and 1 in middle school level.

According to age group of $(7-12)$ the mean of DMFT/dmft was 0.22 /0.55 and mean of Plaque index was 1.27. While in the age group (13 and above) the mean of DMFT/dmft was $0.25 / 0.74$ and the mean of Plaque index was 1.64 (Table 2).

\begin{tabular}{|c|c|c|c|c|}
\hline \multicolumn{2}{|l|}{ Age } & $\mathrm{N}$ & Mean & Std. deviation \\
\hline \multirow{3}{*}{$7-12$} & DMFT & 32 & .2244 & .15475 \\
\hline & $\mathrm{dmft}$ & 28 & .5464 & .31355 \\
\hline & Plaque Index G\&V & 34 & 1.2729 & .37087 \\
\hline \multirow{3}{*}{$=>13$} & DMFT & 28 & .2514 & .11597 \\
\hline & $\mathrm{dmft}$ & 9 & .7433 & .43347 \\
\hline & Plaque Index G\&V & 28 & 1.6432 & .52937 \\
\hline
\end{tabular}

Plaque index revealed that $69.35 \%$ have $>1-2$ soft debris accumulation while $22.58 \%$ have 0 - 1 soft debris accumulation and only $8 \%$ have $>2-3$ soft debris accumulation (Figure 1).

\section{Plaque Index}

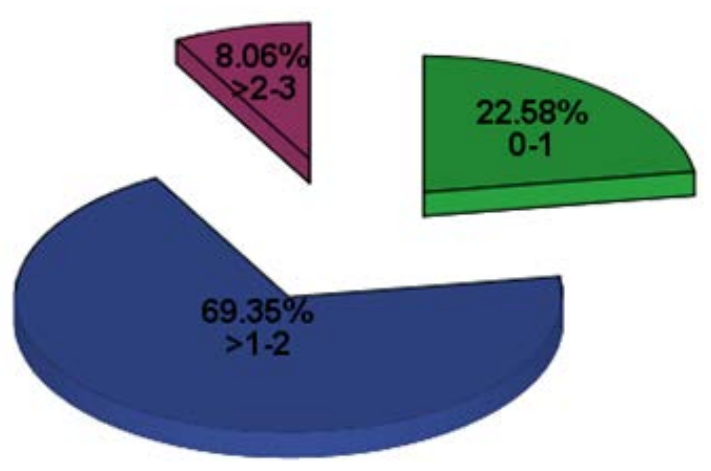

Figure 1.

Interview questionnaire revealed that $87.5 \%$ said brushing will prevent caries, while 9.3\% said No, and 3.1\% they don't know (Figure 2). While 93.76\% revealed that candies will cause caries and $3.1 \%$ said No and 3.1\% they don't know (Figure 3).

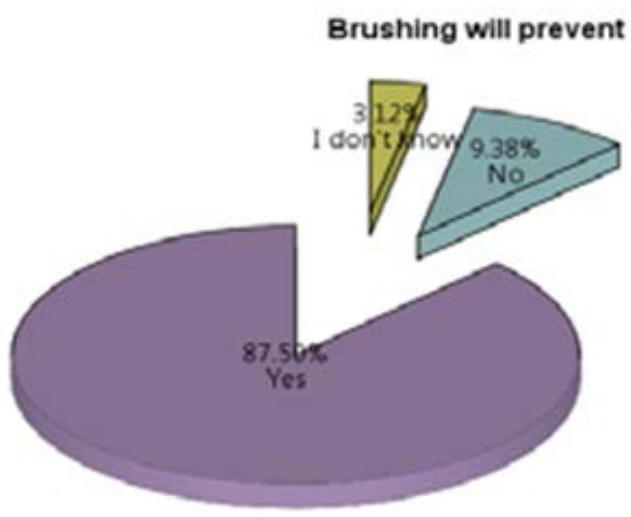

Figure 2.

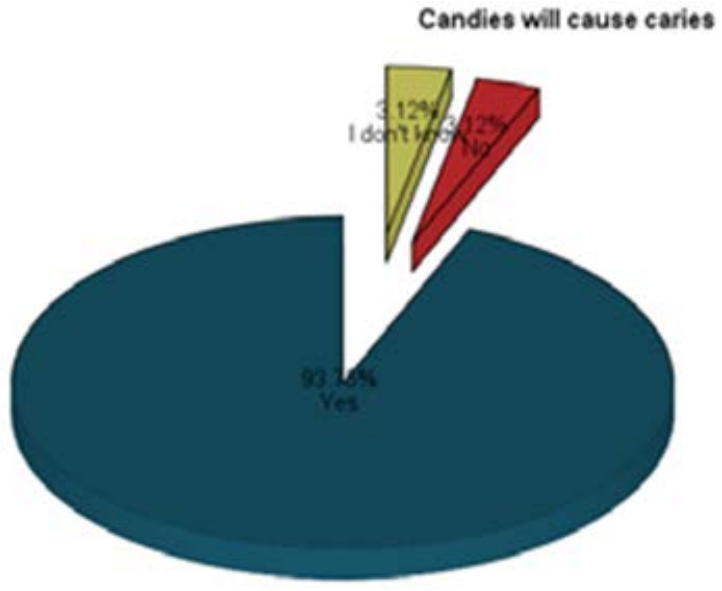

Figure 3.

There was 53\% agree with fluoride benefit for teeth, and $35.9 \%$ they don't know while $10.9 \%$ disagree (Figure 6).

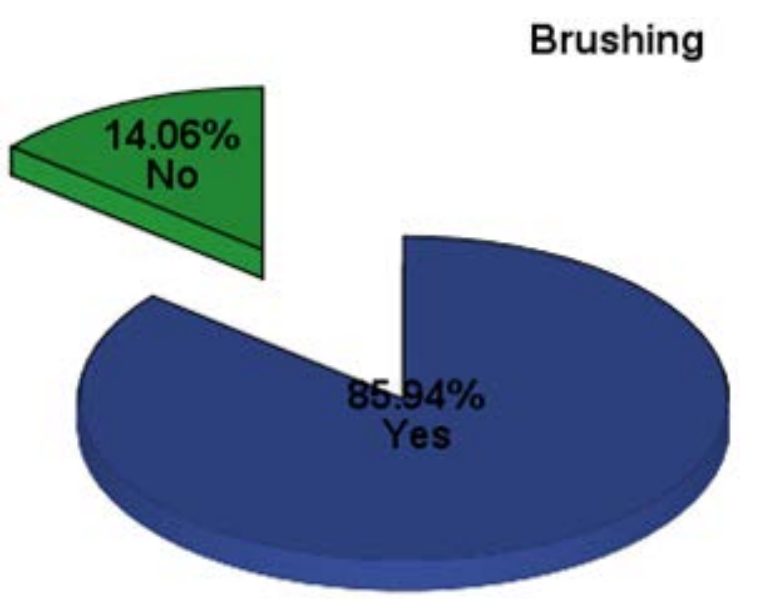

Figure 4.

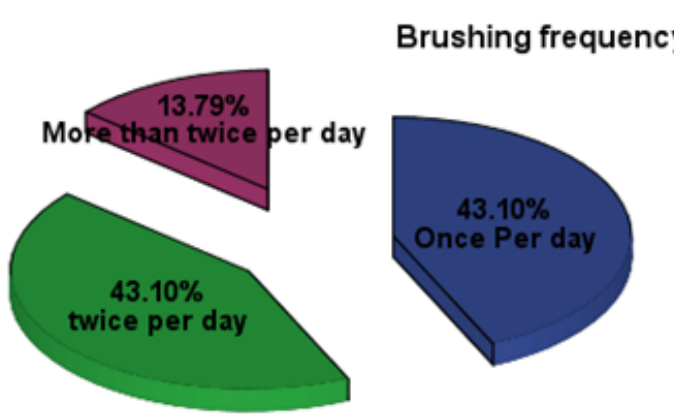

Figure 5.

\section{Fluride benefit for teeth}
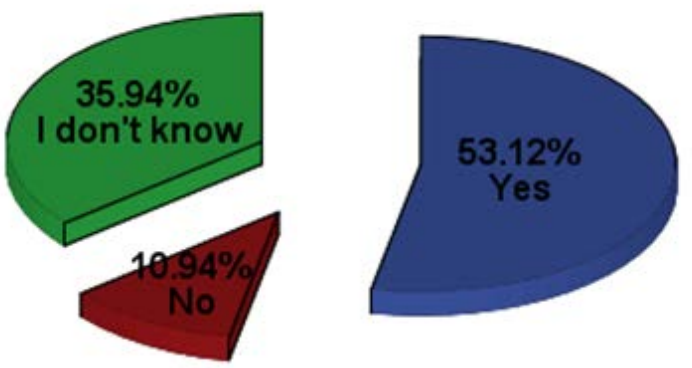

Figure 6.

There was $85.9 \%$ brushing their teeth while $14.1 \%$ they don't (Figure 4). Brushing Frequency revealed that 43.1\% 
brush their teeth once per day and also $43.1 \%$ brush their teeth twice per day while $13.8 \%$ brush more than twice (Figure 5).

Among survey it was found that $48.8 \%$ brush their teeth before sleeping time, and $32 \%$ at morning while $13.7 \%$ after meals and $5 \%$ after eating of candies (Figure 7).

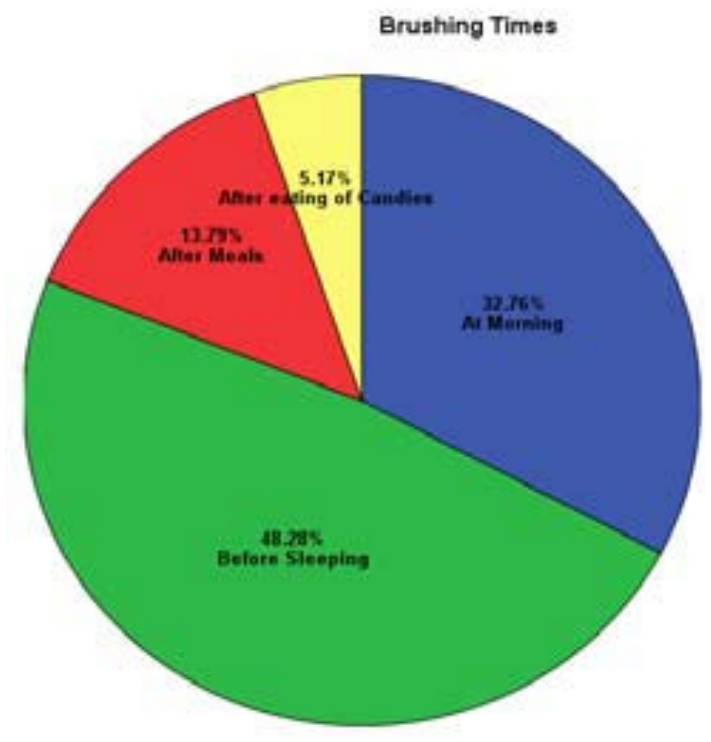

Figure 7.

For the method of brushing it was found that $93 \%$ use tooth brush $3.4 \%$ use fingers and 3.4 use meswak (Figure 8 ).

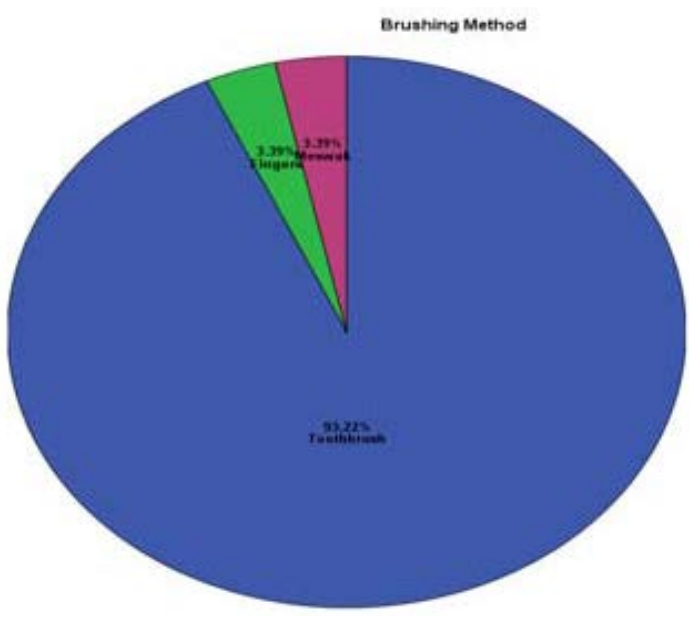

Figure 8.

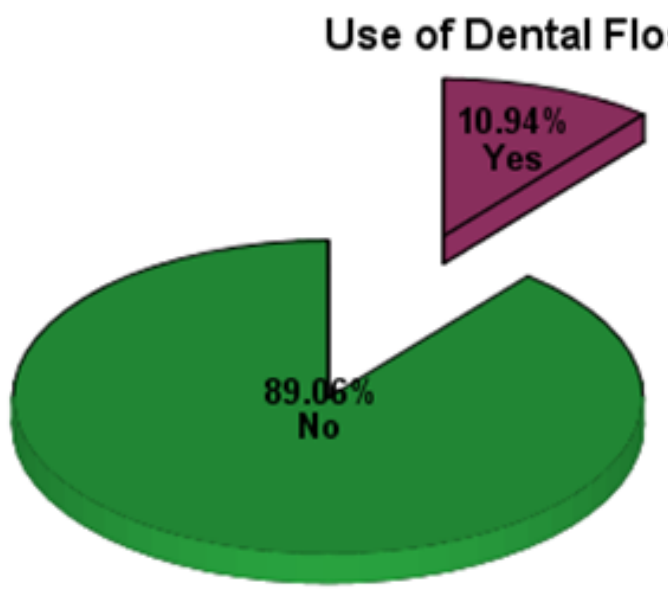

Figure 9.
The interview questionnaire revealed that $62 \%$ they are independent in brushing their teeth without any help, while 17 helped by mothers and $10 \%$ helped by brother \&sister while 6\% helped by father and 3\% helped by others (Figure 9).

Only $10.9 \%$ use dental floss and $89 \%$ never used it (Figure 10).

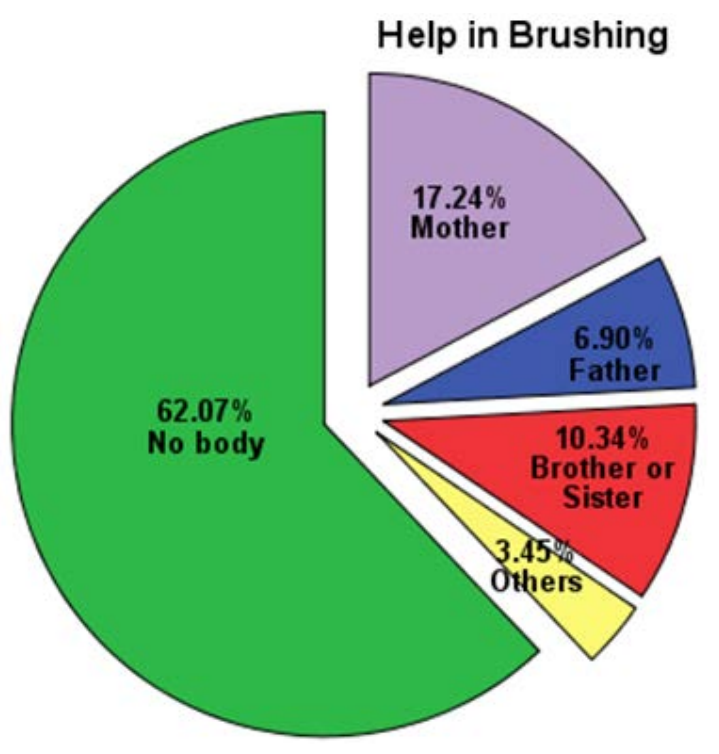

Figure 10.

For the source of brushing education the survey revealed that the majority learned brushing from Home $76 \%$. And 11\% they don't remember while 8.5 learn from schools and 3\% from T.V (Figure 11).

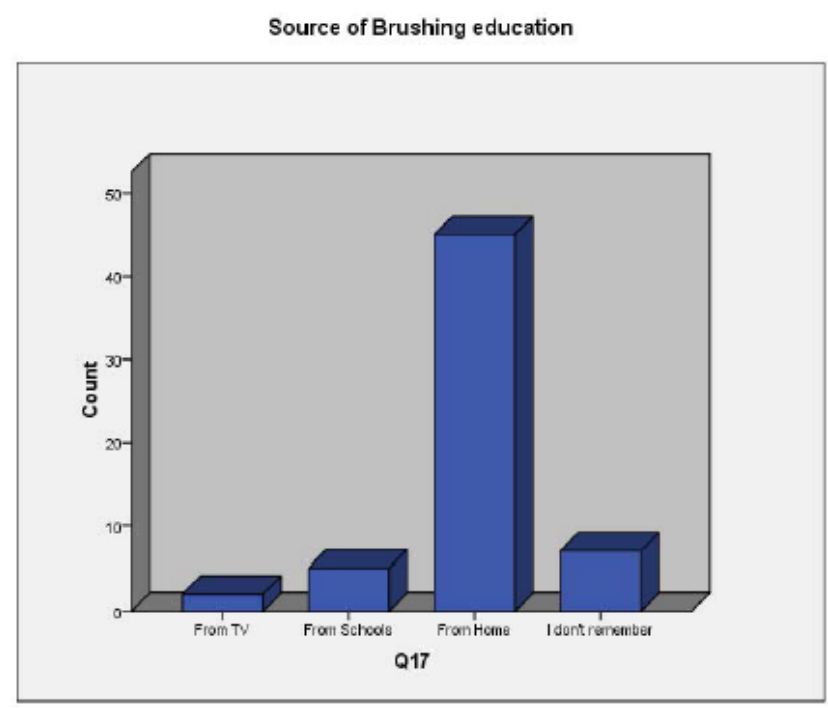

Figure 11.

\section{Discussion}

Dental disease are one of the common problems found in children good oral hygiene is important to all normal children for proper mastication, digestion, appearance, speech and health but it even more important for handicapped children. The process of developing oral disease, prevention and treatment modalities does not differ in disable individual from non- disable. A Study completed by change and shih found that student with 
visual impairment were less knowledgeable about their oral care [4]. In the present study, in contrast to the former, the blind student had better knowledge regarding dental health care, 93\% know the role of sugar in developing dental caries, and $87.5 \%$ know that brushing will prevent caries.

In study conducted by Ahmed et al most of blind students extend having poor oral hygiene total of 80 visually impaired students were examined, of which $44(55 \%)$ were having poor oral hygiene and among them $22.7 \%$ were having dental caries [5].

On the other hand, in a study conducted by ST Prashanth et al they found that $34.1 \%$ of children had dental caries and only $8 \%$ of the blind children have poor oral hygiene. $69.4 \%$ of children had dental caries in deciduous dentition and $35.2 \%$ of children had dental caries in permanent dentition [6].

Goreeleg et al, who did not find any significant relationship between dental decay and blindness among 120 blind students [7].

The present study provide health information on a sample $(n=64)$ of $7-24$ year old visually impaired students and the low DMFT/ 0.24/0.59 results in the present study coincide with that found in Jinan alousi study in Iraq [8].

The reasons for these differences in caries increment may be various, ranging from biochemical differences in salivary buffering to differences in living environment, dietary and hygiene habits, different proportion of salivary component and possible difference in chemical composition of the saliva compared to normal children [9].

This study revealed insignificant relation between neither the mean of DMFT/dmft for both gender Male \& Female $0.24 / 0.57$ and $0.24 / 0.65$ nor the mean of plaque index of both gender 1.5/1.33 respectively. Also the age group distribution revealed insignificant relations.

Plaque index was significant in which $69.35 \%$ have $>1-2$ soft debris accumulation while $22.58 \%$ have $0-1$ soft debris accumulation and only $8 \%$ have $>2-3$ soft debris accumulation, this might because the visually impaired individual cannot visualize the plaque on their tooth, So we need to improve the education modalities to visualize the preventive ways of oral disease and permit for the visually impaired to apply it perfectly.

Among Survey $93.7 \%$ agree with role of sugar in leading to caries while $3.1 \%$ disagree \& 3.1\% they don't know, which coincide with study of Ahmed.M.S et al [5], \& Bhatnagr et al [6]. There is $85.9 \%$ do brushing of their teeth and $14.1 \%$ they don't. There is $87.5 \%$ agree with that brushing will prevent caries but $9.47 \%$ they disagree. For the method of brushing it was found that $93.2 \%$ use tooth brush $3.4 \%$ use fingers and 3.4\% use meswak.

The interview questionnaire revealed that $62 \%$ they are independent in brushing their teeth without any help, while $17 \%$ helped by mothers and $10 \%$ helped by brother \&sister while 6\% helped by father and 3\% helped by others. Only $10.9 \%$ use dental floss and $89 \%$ never used it.
In this study of visually impaired students samples, there was not any significant relationship between dental caries and oral hygiene practice with type of cleaning tools (Toothbrush, Finger and Meswak), use of dentifrices Or, frequency of cleaning (morning, after meals, after eating candies or before sleeping) and neither it was significantly related with knowledge about role of sugar consumption on dental caries nor with important of visiting Dentist. But there is significant dental caries in relation with those help the blind individual in brushing and the use of dental floss.

\section{Conclusion}

The present study shows not more worsening of oral health status in the study population.

A little extra care by the parent or caretaker regarding oral hygiene can give drastic result in reduction of dental caries and gingival disease

\section{Acknowledgements}

Our thanks goes to the director of special education for their permission and without that permission this study would have been impossible task our appreciation also goes to principals of alnoor institute for the blind in Almadinah for their support and cooperation we also want to extend our gratitude to king Fahd specialist dental center for their instrument supply, facilities, and support to continue this research.

\section{References}

[1] MannJ, Wolnerman JS, Lavie G(1984). Periodontal treatment needs and oral hygiene for institutionalized individuals with handicapping condition. sprc care dentistry 1984; 4; 173-176.

[2] Tesini DA (1981). An annotated review of the literature of dental caries and periodontal disease in mentally retarded individuals, Spec Care Dentist.1(2): 75-87.

[3] Zehaati H, Ghandhasi M, Motlagh M. An investigation on DMFT \&DMFS of first permanent molars in 12 yr old blinds in Tehran, iran. J. med.sci. 2006; 6(1); 1-4.

[4] Chang CS, ShihY. Knowledge of dental health and oral hygiene practices of Taiwanese visually impaired and sighted students. $\mathrm{j}$ visual impairment blindness 2004; 98: 1-27.

[5] Ahmed MS, Jindal MK, khan S, Hshmi SH. oral health knowledge, practice, oral hygiene status and dental caries prevalence among visually impaired students in residential institute of aligarah. J Dent oral hygiene2009; 1: 22-6.

[6] ST prashanth, sudhanshu Bhatnagar,usha mohan das, H gopu, oral health knowledge, practice, oral hygiene status, and dental caries prevelance among visually impaired children in Bangalore. J Indian Soc pedo prev Dent 2011; 29: 102-105.

[7] Goreeleg CB, Goldstein PA, Forrester DJ.oral manifestation in a group of blind student. ASDC journal of dentistry for children 1976; 43: 39-41.

[8] Jinan M AL-Alousi.oral health status and treatment needs among blind children in Iraq.Mustansyriah dental journal 2009; 6: 4.

[9] Z.AL-QAHTANI, A.H WYNE. caries experience and oral hygiene status of blind, deaf and mentally retarded female children in Riyadh, Saudi Arabia. Tropical dental journal 2004; 105: 37-40. 